\title{
Interference in a typeface variant of the Stroop test
}

\author{
ROBERT E. WARREN* and MARGOT D. LASHER \\ Columbia University, New York, New York 10027
}

\begin{abstract}
Ss were asked to name the typeface in which a printed item appeared in a discrete trial variant of the Stroop color-word test. Two kinds of items were used: (a) typeface names appearing in antagonistic typefaces, e.g., the word SCRIPT printed in bold type; and (b) nonsense strings constructed by jumbling the letters in a typeface name, e.g., PSRTCI printed in bold type. Typeface-naming latencies were found to be significantly longer for items of the first kind. Examination of the distributions of individual trial latencies for the two kinds of items indicated that modification of Morton's (1969) account of the Stroop test is required.
\end{abstract}

In the traditional version of the Stroop test (Stroop, 1935), Ss are run through a series of three tasks: (a) reading aloud a series of color names printed in black ink; (b) naming the colors of a series of color patches; and (c) naming the colors in which a series of color names are printed. In the last task, the color names are printed in antagonistic colors of ink, e.g., the word YELLOW printed in blue ink. In general, investigators have found that the time to perform the three tasks varies, that for the first task being the shortest while the second and third tasks take progressively longer (Jensen \& Rohwer, 1966).

This result has been interpreted in various ways. Stroop (1935) believed that competition between two responses-the word-reading response and the color-naming response-was involved. Although the exact mechanism of this competition remained unspecified, it was assumed that the word-reading response was in some sense stronger than that for color naming and so interfered when the latter was the task to be performed. This difference in strength is reflected in the difference between the time needed to name a series of color patches and that required to read an equal number of color names. This result parallels a similar difference in the time needed to name objects and to read their names (Cattell, 1886; Oldfield, 1966).

Morton (1969) has proposed an explanation of the Stroop effect in terms of what he calls a logogen model. In this model each word in a S's vocabulary is represented by a word recognition unit or logogen. This unit is composed of three elements: (a) a counter that increments in value each time stimulus information that corresponds to the word the logogen represents is encountered; (b) a motor program that represents the verbal production of the word; and (c) a threshold value that is fixed by the past frequency of occurrence of the word. Upon presentation of a word, counters at logogens which correspond in whole or part to the physical

\footnotetext{
*Requests for reprints should be directed to R. E. Warren, Department of Psychology, Columbia University, New York, New York 10027.
}

representation of the word begin to increment, those which correspond best increasing in value at the greatest rate. When one of the logogens' counter values exceeds threshold, its motor program is said to become available and is loaded into a response buffer for output. Only one program can be loaded into the buffer at a time. Presentation of a word, then, increases the count at the logogen corresponding to it. This activation process is seen as temporary, with the count at the logogen declining gradually as a function of time since presentation.

Interference in the Stroop situation, according to this model, occurs because stimulus information strongly activating two logogens is presented simultaneously. When the word BLUE is shown in green ink, for example, both the logogen for BLUE and the logogen for GREEN should be activated. Since only one motor program can be loaded into the response buffer at a time, one of the programs is delayed. On a certain proportion of trials the program for the base word is loaded first, and it is the ink color name program that is blocked. On these trials, the ink color name program is delayed until the buffer can be cleared and an increase in color-naming latency is observed.

The chief advantage of Morton's model is heuristic: it relates the Strocp situation to a number of other, quite different, experimental paradigms. The model leaves several questions unanswered, however. First, the lack of any significant interference with word reading when the words are color names printed in antagonistic ink colors (Stroop, 1935) implies an asymmetry in the tasks of color naming and word reading. One possible explanation for this discrepancy is that color naming includes the extra step of color identification before there is any input to the logogen system, while words have direct access. A second problem is more important: In the interference situation, that is, where a color name appears in an antagonistic ink color, why is it ever the case that the $\mathbf{S}$ produces a correct response? Since the base word is itself a color name, a simple check against a list of permissible responses is not sufficient. What is 
necessary is some secondary check on the source of the response information, an addition which negates some of the simplicity of the model. Finally, the model appears to predict a probabilistic interference function. That is, for that proportion of trials on which the base word is loaded into the response buffer first, there should be long color-naming latencies. On the remaining trials, those on which the ink color name is loaded in the buffer first, there should be no delay relative to simple color patch naming. This interference function could be expected to produce a bimodal distribution of color-naming latencies when the base item was an antagonistic color name, with the two modes of the distribution corresponding to the two buffer-loading outcomes mentioned. Barring actual bimodality, one would expect at least an increase in the variability of response latencies over that found for color naming when the base item was simply a patch of color.

White (1969) has recently suggested that color-naming interference is not a unique situation and that, indeed, any task which requires Ss to respond to an attribute of a printed or spoken word aside from its name will show similar effects. The present experiment utilizes the typeface in which a word is printed as the attribute corresponding to color in the Stroop test. By utilizing a discrete trial version of the Stroop, instead of the typical serial presentation, it is hoped that a test of the third question raised about Morton's model can be made.

\section{METHOD}

\section{Subjects}

Thirteen male and seven female students recruited from the undergraduate population at Columbia University served as Ss. Ss were run individually for a single $1 / 2-\mathrm{h}$ session and received $\$ 1.75$ for their participation.

\section{Procedure}

Ss were seated in a darkened room in front of a rear projection screen on which all the stimuli in the experiment were shown. A Gerbrands projection tachistoscope was used to present the stimuli.

On each trial in the experiment an item printed in one of four typefaces was presented. Items were printed in white letters and centered on a gray background. Ss were instructed to call out the name of the typeface in which an item was printed as quickly as possible and to ignore the item itself. Naming the typeface activated a voice-operated relay which terminated the visual display and stopped a millisecond clock which had been started at item onset. There were $7 \mathrm{sec}$ between trial onsets. Naming latency and accuracy were recorded.

\section{Design and Materials}

The four typefaces used in the experiment were designated as script, modern, English, and bold. Stimuli were prepared using Letraset transfer type lowercase fonts of Palace Script, Optima, Old English, and Franklin Gothic, respectively. Items containing the same number of letters were approximately the same length in all typefaces. The items used subtended an average visual angle of $4.3 \mathrm{deg}$.

Two sets of stimuli were prepared, a practice set and an experimental set. In the practice set the items appearing in type were of two varieties: (a) the name of a typeface printed in the same typeface, e.g., the word SCRIPT printed in script type; (b) a nonsense string constructed by jumbling the letters of a typeface name, e g., PSRTCI printed in script type. There were also two kinds of items in the experimental set: (a) test items consisting of a typeface name printed in an antagonistic typeface, e.g., the word BOLD printed in modern type; (b) control items consisting of nonsense strings constructed by jumbling the letters of a typeface name, e.g., DNEMRO printed in bold type. All the nonsense string items in the experimental set were different combinations of letters and typefaces than were used in the practice set.

At the beginning of the experiment, Ss were shown one example of each typeface using items drawn from Practice Set Variety A. They were then run through a block of 24 trials using equal numbers of items of both practice set varieties. Items were ordered randomly in this block with the restriction that the same typeface-naming response should not occur on successive trials. Following the practice trials, Ss were run through a block of 100 experimental trials. The first four trials in this block were drawn from Practice Set Variety B and served as filler trials. Items used on the remaining 96 trials were drawn from the experimental set. To counterbalance practice effects, the 96 trials were divided into four 24-trial groups. Within each group, each of the experimental items appeared once and half of the trials were test trials and half control trials. Each typeface was used on an equal number of test and control trials. Within the four groups, trials were ordered randomly with the restriction that neither the same typeface name nor typeface-naming response occur on successive trials.

\section{RESULTS}

Typeface-naming accuracy was quite good, only $3.7 \%$ of the possible errors occurring. Trials on which typeface-naming errors occurred were discarded from further analysis.

Mean naming latencies for the test and control conditions were determined for each $\mathrm{S}$. Overall means for the test and control conditions were 1,053 and $1,016 \mathrm{msec}$, respectively. A repeated measures analysis of variance was done with condition (test, control) and Ss as factors. The main effect for condition was significant, $F(1,19)=6.26, p<.025$.

Individual trial latency distributions for the test and control conditions were also determined and are shown in Fig. 1. Both distributions appear to be unimodal, the test distribution being shifted slightly higher than the control distribution. Variances for the test and control distributions were $82,857.56$ and $82,394.05$, respectively. The difference between the two variances was not significant, $F(917,932)=1.01, \mathrm{p}>.05$.

\section{DISCUSSION}

The results of the experiment appear to confirm that interference effects similar to those observed in the Stroop color-naming task are produced when Ss are asked to name the typeface in which an antagonistic typeface name is printed. Along with other studies (White, 1969: Morton, 1969), the present findings indicate that the color-color name combination is not unique in producing interference. It appears that any task in which the $\mathrm{S}$ is asked to report a characteristic of 
the physical representation of a word can be disrupted by the identity of the word itself. This seems to hold true for auditory characteristics of spoken words as well as visual attributes of printed words (Bever \& Mansfield, 1973).

The experimental outcome casts some doubt on the probabilistic interference function predicted from the logogen model. If the base word is loaded into the response buffer on only a certain proportion of the trials in the test condition, then one would expect a bimodal distribution of naming latencies or at least an increase in the variability of the distribution. That is, test trials where the base word was loaded into the buffer first and subsequently cleared should have produced distinctly longer naming latencies, while those test trials on which the typeface name was loaded into the buffer first should have produced latencies indistinguishable from those found in the control condition. Contrary to this prediction, the distributions of test and control latencies seem to be identical in form and variance, with the test distribution having a slightly higher mean.

To account for this result, one might assume that the base word in the typeface-naming task is loaded into the buffer first on every trial and rejected only upon completion of the source check mentioned earlier. Control trials would never produce this situation, since the nonsense strings used as carriers for the typeface do not correspond to any logogen and so never produce a motor program before the typeface is loaded into the buffer. Thus, latencies on all trials in the test condition would be increased over those in the control condition by an equal amount.

How could this situation arise? A reexamination of the assumptions of the logogen model provides a possible explanation. First, the model assumes that the activation at a logogen persists for some time after the presentation of a word before returning to zero. Since the typeface names occur constantly during the naming task, there is likely to be some degree of residual activation at any given typeface name logogen when that name occurs as a base item. The time needed for the logogen counter to pass threshold is therefore greatly reduced, and the base word logogen's motor program is loaded into the response buffer more quickly than in the case of an unactivated logogen. This decreased loading latency alone, however, cannot assure that it will be the base word's logogen that will enter the buffer first, since the residual activation at typeface name logogens should provide an equal benefit to the typeface identity response.

To predict the loading priority necessary to account for the results, one must also assume that the base word has more direct access to the logogen system than the typeface identity. This assumption corresponds to the resolution offered earlier to the word-reading color-naming asymmetry problem. The reading-naming asymmetry presumably occurs because the color identity

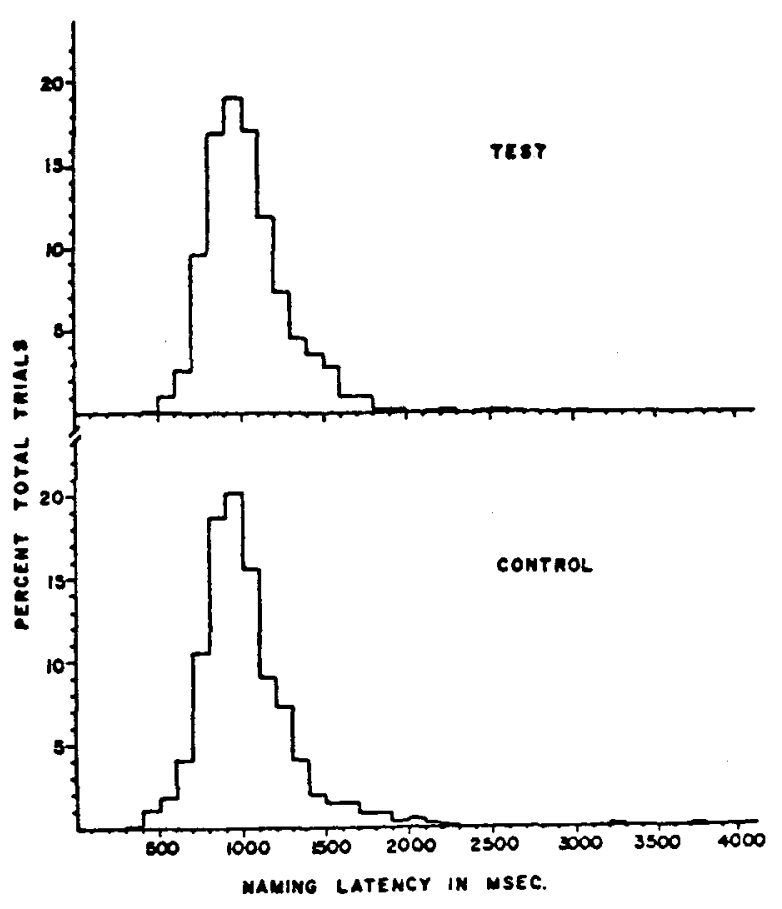

Fig. 1. Individual trial typeface-naming latency distributions.

input to the logogen system lags behind that provided by the base word and so never blocks access of the base word motor program to the response buffer. Similarly, the base word program is always loaded into the buffer first in the typeface-naming task because the typeface identity input to the logogen system is delayed and so does not profit equally from the residual activation at the typeface name logogens. In both cases, the delay of input is presumably produced by an extra object or feature identification step required before any input to the logogen system about color or typeface identity is possible.

\section{REFERENCES}

Bever, T. G., \& Mansfield, A. Verbal Stroop and lexical associations in children and adults. Unpublished manuscript, Columbia University, 1973.

Cattell, J. McK. The time it takes to see and name objects. Mind, $1886,11,63-65$.

Jensen, A. R., \& Rohwer, W. D., Jr. The Stroop color-word test: A review. Acta Psychologica, 1966, 25, 36-93.

Morton, J. Categories of interference: Verbal mediation and conflict in card sorting. British Journal of Psychology, 1969, 60, 329-346.

Oldfield, R. C. Things, words and the brain. Quarterly Journal of Experimental Psychology, 1966, 18, 340-353.

Stroop, J. R. Studies of interference in serial verbal reactions. Journal of Experimental Psychology, 1935, 18, 643-662.

White, B. W. Interference in identifying attributes and attribute names. Perception \& Psychophysics, 1969, 6, 166-168.

(Received for publication May 11, 1973; revision received August 27, 1973.) 